ISSN 2447-9071

doi https://doi.org/10.36414/rbmc.v7i17.87
Contato para correspondência: Kauara Vilarinho Santana Vieira

E-mail:

kauaravvieira@gmail.com

Conflito de interesse: Não

Financiamento: Recursos próprios

\title{
A importância da fisioterapia na capacidade funcional de idosos com risco de quedas
}

\section{The importance of physiotherapy in the functional capacity of elderly people at risk of falls}

Stéfanny de Liz Sofiatti', Mirelly Mendes de Oliveira', Lorraine Moura Gomes' ${ }^{1}$, Kauara

Vilarinho Santana Vieira'

${ }^{1}$ Faculdade UniBRAS

\begin{abstract}
Resumo
O presente estudo teve como objetivo destacar a importância da fisioterapia na prevenção e no tratamento das quedas em pacientes idosos, visando a sua melhoria de qualidade de vida e maior capacidade funcional. Para tanto descrevemos resumidamente sobre a incidência das quedas, seus fatores de risco intrínsecos e extrínsecos e as manifestações clínicas que elas geram nos idosos. Tratase de uma revisão bibliográfica narrativa, na qual os artigos foram acessados por meio das bases de dados publicadas entre 2010 e2020. Foram selecionados 42 artigos e 4 literaturas clássicas. De acordo como estudo feito conclui-se que o fisioterapeuta deve ser um profissional capacitado para atuarnão só no tratamento, mas também na prevenção, pois essa assistência multifatorial reduz a chance de quedas. Sendo assim o fisioterapeuta é detentor de conhecimentos necessários para orientar tanto os pacientes quanto seus familiares, com formas seguras de prevenir os riscos de quedas, além de trabalhar com o idoso, formas de aumentar sua capacidade funcional, equilibrio, suporte muscular e sua independência, podendo realizar suas atividades de vida diária com segurança e bem-estar.

Palavras-Chave: Capacidade Funcional; Quedas; Fisioterapia Geriátrica.
\end{abstract}

\begin{abstract}
The presentstudy had as goal to highlight the importance of the physical therapyin the prevention and treatment of the elderly, aiming the improvement of their quality of life and better functional capacity. Therefore, it is briefly reported about the incidence of the falls, theiroutward and intrinsic riskfactors, and the clinical manifestation that they can cause in the elderly. It is about an integrative literature review, in which the articles were accessed through the database between 2010 and 2020. Forty-two articles and twenty classic literature were selected. According to the study, it can be concluded that the physical therapist must be a professional skilled to act not only on the treatment but also on the prevention, because this multifactorial assistance reduces the chances of falls, and knows how to guide not only the patient but also its family, with secure ways to prevent the risk of falls, besides working with the elderly, ways of increasing their functional capacity, balance and muscle support and independence, so they can accomplish their daily activities with security and well-being.
\end{abstract}

Keywords: Functional capacity; Falls, Geriatric Physiotherapy. 


\section{Introdução}

O envelhecimento populacional atualmente é um fenômeno universal, tanto em países desenvolvidos como em emergentes igual ao Brasil e, para tal, tem recebido especial atenção no que diz respeito à políticas que viabilizem não só a possibilidade de as pessoas viverem mais anos, mas que esses sejam de qualidade de vida no que diz respeito aos aspectos biopsicossociais ${ }^{1,2}$.

A velhice, ou a terceira idade é uma fase da vida onde a pessoa tem alterações que levam à diminuição de força e degeneração do organismo, o que acarreta diversos acidentes, dentre eles, o mais comum, a queda. A fisioterapia tem papel fundamental nesta etapa, para um envelhecimento ativo, tentando preservar a capacidade funcional do idoso para prevenir os constantes riscos de queda e dar a ele independência e qualidade de vida ${ }^{3,4}$.

O conceito de envelhecimento ativo, tal como proposto pela Organização Mundial de Saúde apoia-se nos pilares: vida saudável, vida participativa, seguridade social e educação permanente. Para promover o"envelhecimento ativo" de nossa população será necessário, portanto, promover essa mesma concepção como objeto de interesse de estudo e de propostas de políticas públicas, bem como será necessário promover a compreensão desse fenômeno chamado de envelhecimento ${ }^{5,6}$.

Alterações fisiológicas próprias da idade podem levar a uma atrofia e fraqueza muscular maior, a sarcopenia. As alterações ósseas e musculares observadas com o aumento da idade são interdependentes. Ossos mais frágeis são apoiados por músculos mais fracos, o que leva a quedas e à sua consequência mais temida, as fraturas ${ }^{7}$.

As alterações fisiológicas intrínsecas ao envelhecimento são sutis, inaptas a gerar qualquer incapacidade na fase inicial, embora, com o passar dos anos, venham a causar níveis crescentes de limitações ao desempenho de atividades básicas do cotidiano. A visão é um processo complexo que envolve a integração das estruturas do olho, o controle motor e o controle neural. Sua importância reside na obtenção de informações sobre o ambiente e na orientação do movimento do corpo. Com o envelhecimento, o enfraquecimento do sistema visual favorece a ocorrência de quedas ${ }^{7,8}$.

A redução da flexibilidade de membros inferiores também pode determinar risco de quedas. A literatura encontrada enfatiza que a diminuição da amplitude de movimento e força muscular nos movimentos de forma geral, principalmente em membros inferiores, geram alterações nos padrões de marcha e dificuldades no desempenho de atividades do cotidiano, associando à ocorrência de quedas ${ }^{9,10}$.

O fisioterapeuta em sua formação tem conhecimentos específicos para a atenção ao idoso, pois tem conhecimento das alterações fisiológicas do envelhecimento nos níveis anatômicos e fisiológicos, ajudando na prevenção e no tratamento, trabalhando o equilíbrio, devolvendo ao paciente a qualidade de vida e a autonomia do mesmo. Cabe então aos profissionais da área se atentarem para este cenário, cada qual atuando em suas especificidades, e todos colaborando para a melhor condição de vida dos indivíduos idosos, orientando o familiar responsável e o idoso com formas seguras para evitar quedas $^{3-11}$.

No processo de envelhecimento, a maioria dos gestos motores tornam-se cada vez menos seguros, entre os quais se encontram a realização de atividades de vida diária (AVD's). As funções locomotoras, sensoriais e cognitivas estão intrinsecamente relacionadas com a mobilidade. Aproximadamente $20 \%$ da população, que se encontra em processo de envelhecimento, caem a cada ano. As quedas podem resultar em fraturas e causar sérias consequências, como lesões permanentes, perturbação na mobilidade, declínio funcional e posterior internação em asilo, além de ser considerada a principal causa de eventos traumáticos em idosos representando $90 \%$ dos atendimentos hospitalares desta população-12.

O estudo tem o objetivo de fazer uma revisão de literatura sobre os principais meios de prevenir e/ou minimizar riscos de quedas, orientar a família e o idoso sobre o ambiente em que vive; identificar as consequências do idoso devido aos fatores de riscos das quedas e analisar as principais intervenções de quedas das pessoas idosas, pois, tendo o paciente sua capacidade funcional preservada, ele terá uma melhor qualidade de vida.

\section{Métodos}

Trata-se de uma revisão narrativa da literatura. Este tipo de revisão são publicações apropriadas para descrever e discutir o desenvolvimento de um determinado tema, sob o ponto de vista teórico. São análises de livros e artigos científicos, impressos ou digitais, na interpretação crítica do autor. As revisões narrativas permitem ao leitor atualizar seus conhecimentos sobre um assunto específico em um espaço de tempo menor.

O levantamento bibliográfico foi realizado em bases eletrônicas e busca em literaturas clássicas. As bases de dados pesquisadas foram: Medical Literatura Analysis an Retrieval System Online (MEDLINE), Scientific Eletronic Library Online (SCIELO), no US National Library go Medicine (PubMed) e periódicos da Coordenação de Aperfeiçoamento de Pessoal de Nível Superior (CAPES). O período de abrangência foi entre janeiro de 2010 a junho de 2020.

Os descritores utilizados foram os padronizados em Ciências da Saúde: capacidade funcional, quedas, idosos, fisiote- 
rapia e geriatria. A fim de especificar ainda mais a busca dos artigos, foram acrescentados qualidade de vida, prevenção, prognóstico, independência funcional.

Iniciou-se a leitura dos resumos de todos os artigos encontrados a fim de fazer uma seleção mais específica, e para aqueles que enquadravam no perfil do artigo, fez-se a leitura integral do mesmo. Após a leitura dos artigos selecionados, os mesmo foram separados de acordo com os assuntos abordados afim, de um direcionamento melhor durante a construção da revisão.

Os critérios utilizados para essa seleção foram: serem artigos científicos, estudos de caso e revisões sistemáticas sobre o tema quedas de idosos, capacidade funcional após quedas, abordagens fisioterapêuticas em quedas, prevenções para quedas, prognóstico, principais causas de quedas em idosos, fatores intrínsecos e extrínsecos, independência funcional na terceira idade, importância da fisioterapia.

\section{Resultados e Discussão}

\section{Os riscos de queda e sua incidência}

O envelhecimento, que inexoravelmente acomete o indivíduo com o passar dos anos, interfere nos seus diversos aparelhos e sistemas orgânicos. Alterações funcionais daí decorrentes produzem diferentes consequências para o idoso, como por exemplo, distúrbios na marcha. A marcha é resultante da interação dos sistemas neurológico, musculoesquelético, vestibular e somato-sensorial. As quedas na população idosa são frequentes e determinam complicações múltiplas que afetam negativamente a qualidade de vida dessas pessoas ${ }^{13,14}$.

A queda pode ser considerada um marcador do início de um importante declínio de determinada função ou um sintoma de uma patologia nova. A conceituação de quedas pode ser entendida como uma insuficiência súbita do controle postural uma falta de capacidade para corrigir o deslocamento do corpo, durante seu movimento no espaço; uma mudança de posição inesperada, não intencional, que faz com que o indivíduo permaneça em um nível inferior; bem como um deslocamento não intencional do corpo para um nível inferior em relação à posição inicial ${ }^{15,16}$.

O número de quedas aumenta em proporção ao aumento da população idosa no mundo, elas aumentam exponencialmente com as alterações biológicas relacionadas com à idade. Assim sendo, um número significativo de pessoas com idade superior a 80 anos apresenta um aumento substancial de quedas com lesões associadas. Os indivíduos com idade superior a 65 anos apresentam uma incidência de 28 a 35\% de quedas anualmente. Os indivíduos com idade superior a 70 anos apresentam um aumento da incidência para 32 a $42 \%{ }^{17,18}$.

\section{Consequências das quedas em idosos}

As consequências não são somente físicas, mas psicológicas e sociais como hospitalização institucionalização ou reabilitação, diminuindo autonomia e aumentando custos para a saúde pública. Os idosos restringem suas atividades devido a dores, incapacidades, medo de cair, atitudes protetoras de familiares e cuidadores ou até mesmo por aconselhamento de profissionais de saúde ${ }^{1,19}$.

As fraturas são uma das consequências mais comuns entre idosos após uma queda, principalmente as fraturas de quadril, seguidas pelas de punho. $O$ medo de cair tem consequências negativas no bem-estar físico e funcional dos idosos, no grau de perda de independência, na capacidade de realizar normalmente as atividades de vida diária (AVD's) e na restrição da atividade física, explicando o grau de prevalência do estilo de vida sedentário nos idosos ${ }^{15-20}$.

\section{Fatores de risco intrínsecos e extrínsecos para as quedas}

Os fatores intrínsecos englobam as alterações visuais, alterações cognitivas, alterações musculoesqueléticas, déficit vitamínico, iatrogenia, patologia cardiovascular, deformidades nos pés e comorbidades que o idoso apresenta, como diabetes mellitus ou depressão. Os fatores extrínsecos abrangem as características do meio envolvente e o uso de calçado inadequado ${ }^{17-21}$.

Com o envelhecimento, o sistema visual passa por inúmeras modificações, tanto nas funções visuais quanto na visão funcional. Dentre as alterações das funções visuais pode-se destacar: acuidade visual, campo visual, sensibilidade ao contraste, percepção de profundidade e absorção de luz. Além das alterações próprias do envelhecimento, as patologias oftalmológicas como catarata e glaucoma são comuns nos idosos e as duas interferem diretamente na visão funcional dos mesmos, pois reduzem a capacidade de enxergar com clareza e nitidez durante as atividades cotidianas, aumentando assim o risco de quedas ${ }^{17-22}$.

A força muscular é necessária para a performance ajustada das atividades de vida diária. A diminuição da potência muscular influencia a reação postural a perturbações externas, resultando em diminuição do equilíbrio e, consequentemente, em queda. Doenças sistêmicas, como por exemplo, a Diabetes Mellitus (DM), em idosos estão intimamente associadas ao desequilíbrio e quedas, pois provocam a diminuição da função sensoriomotora ${ }^{17-23}$.

As quedas poderão ser vistas como consequência da função neuromuscular deficitária associada à défice de vitamina $D$, uma vez que a performance motora anormal, o aumento do desequilíbrio postural e a fraqueza do quadríceps femoral 
foram verificados em indivíduos com baixo status de vitamina D. Os medicamentos que aumentam o risco de queda englobam vários psicotrópicos como sedativos, hipnóticos e antidepressivos, que podem afetar a atenção, equilíbrio, movimento e regulação da pressão arterial, associando-se à ocorrência de quedas ${ }^{17-24}$.

Idosos que caem e perdem a consciência têm maior probabilidade de ter uma causa cardíaca subjacente do que indivíduos com idade inferior a 60 anos. Um estudo realizado demonstrou que a redução da flexibilidade do tornozelo, a deformidade do hálux valgus, a diminuição da sensibilidade plantar táctil e força do flexor plantar do pé, bem como a existência de dor nos pés estão associadas a quedas ${ }^{17-25}$.

Os fatores extrínsecos que estão relacionados aos comportamentos e atividades das pessoas idosas e ao meio ambiente são: ambientes inseguros, mal iluminados, planejados e construídos, com barreiras arquitetônicas, representam os principais fatores de risco para quedas. Fatores extrínsecos: pisos escorregadios, tapetes soltos, escadas sem corrimão, iluminação inadequada, interruptores em locais inadequados moveis inadequados e mal instalados e calçados inadequados ${ }^{12,26}$.

Extrínsecos (destacam-se os perigos ambientais e sociais que desafiam o idoso) que influenciam na incidência de quedas entre os idosos, sendo que os principais fatores de risco para quedas nesse público são idades avançada. Alguns idosos, por não aceitarem suas limitações, se colocam em situações de risco, realizando atividades que resultam em quedas, como por exemplo: subir em uma escada dobrável para limpar um armário ou para pegar algum utensílio doméstico ${ }^{12,27,28}$.

A identificação dos fatores de risco causadores das quedas é uma importante estratégia para estabelecer programas de prevenção das mesmas. Um ambiente pode ser considerado ideal para o idoso quando oferece segurança, facilita o desenvolvimento da sua funcionalidade, proporciona a estimulação cognitiva, facilita a mobilidade e a interação social, favorece a adaptação às mudanças sendo aconchegante e familiar para o idoso ${ }^{28-29}$.

É importante ressaltar que as mudanças realizadas no domicílio devem ser realizadas com a autorização do idoso, pois sua casa e seus objetos possuem uma memória afetiva e qualquer alteração irá necessitar de uma reorganização interior para fornecer maior segurança ${ }^{28,30}$.

\section{Fisioterapia no tratamento dos acometimentos gerados pelas quedas}

A capacidade funcional é definida como a habilidade de manter as atividades físicas e mentais necessárias ao idoso, o que significa poder viver sem ajuda para as atividades básicas e instrumentais da vida diária. Esse comprometimento tem implicações importantes para o idoso, sua família, a comunidade e o sistema de saúde, uma vez que a incapacidade ocasiona maior vulnerabilidade e dependência na velhice ${ }^{31,32}$.

Ao tratar uma consequência decorrente da queda, o profissional precisa direcionar seu olhar não apenas para as sequelas físicas, mas também, para o quanto a queda pode interferir nas atividades cotidianas do idoso. É preciso avaliar, a partir do primeiro atendimento, a dimensão do evento, o quanto ele interferiu e pode interferir na capacidade funcional da pessoa. Quanto mais precocemente forem tratadas as incapacidades em decorrência da queda, as complicações mais graves podem ser prevenidas ${ }^{31,33}$. Nessa abordagem, a fisioterapia procura restabelecer e melhorar a capacidade funcional dos idosos, prevenindo seu agravamento. Seu enfoque será avaliar o indivíduo como um todo, seu sistema musculoesquelético, neurológico, urológico, cardiovascular e respiratório, assim como o meio em que vive, e identificar as pessoas que acompanham este idoso, bem como suas relações sociais ${ }^{9,34}$.

O fisioterapeuta organizará uma proposta de promoção da saúde ao idoso a partir da observação realizada previamente, partindo das alterações encontradas. Deve-se considerar nessa abordagem, a percepção desse idoso em relação às atividades básicas diárias, assim como às instrumentais, e procurar contextualizá-las junto à realidade, preservando sua individualidade, subjetividade e cotidiano ${ }^{9,35}$.

Estudos que associaram componentes de força e/ou equilíbrio, além de outras formas de intervenção, que tenham sido realizados, no mínimo, duas vezes por semana e que tenham acompanhado os indivíduos, em média, de 3 a 6 meses após a intervenção, mostraram-se efetivos em reduzir e prevenir as quedas em idosos ${ }^{36,37}$.

O treinamento do equilíbrio foi associado ao treinamento de força e com programa de fisioterapia convencional, também incluíram exercícios de equilíbrio que foram associados a diferenciados tipos de exercícios (alongamento, força, aeróbicos e de coordenação motora). O Tai Chi também foi utilizado no protocolo de tratamento ${ }^{36,38}$.

A hidroterapia é eficiente para a melhora do equilíbrio postural para idosos, tanto durante a realização de alguns movimentos que simulam atividades funcionais como durante a marcha. Os benefícios da fisioterapia aquática propõem, dessa forma, protocolos de tratamento no qual o idoso poderá realizar os exercícios de reabilitação sem riscos de quedas ou novas lesões, em um ambiente agradável que permite a realização de exercícios que em terra seriam dificultados ou impossíveis ${ }^{39}$. 
Com base em estudos que mostram efetividade no protocolo de tratamento avaliado, a hidroterapia e cinesioterapia apresentam bons resultados na reabilitação de idosos com histórico de quedas, são efetivas para a melhora do equilíbrio e agilidade, amenizando o declínio da capacidade funcional inerente ao envelhecimento. Ainda, a prática de exercício físico, além de combater o sedentarismo, contribui de maneira significativa para a manutenção da aptidão física do idoso $0^{39,40}$.

Os protocolos de hidroterapia usados no tratamento englobam exercícios com: alongamento dos músculos isquiotibiais; alongamento dos músculos tríceps sural e iliopsoas; flexoextensão de ombros bilateral, associando a inspiração na flexão e expiração na extensão; abdução-adução horizontal dos ombros bilateral, associando a inspiração na abdução e expiração na adução; controle respiratório, associado a uma extensão de tronco; marcha em fila, deslocando-se na piscina; marcha com paradas em apoio unipodal; marcha com um pé à frente do outro; bombeamento de tornozelo, realizando extensão dos joelhos associada à flexão plantar; todos realizados na piscina aquecida ${ }^{38,40}$.

Já os protocolos de cinesioterapia trabalham exercícios com: controle respiratório sentado na bolsa suíça; alongamento dos músculos isquiotibiais; alongamento dos músculos tríceps sural e iliopsoas; abdução-adução horizontal dos ombros bilateral; marcha com paradas em apoio unipodal; expansão torácica em decúbito dorsal; fortalecimento dos eretores da torácica; treino de equilíbrio em superfície instável como a prancha de equilíbrio e marcha com obstáculos no chão. ${ }^{40-41}$

\section{Fisioterapia na prevenção de quedas}

A prevenção de quedas em qualquer ambiente ou contexto é desafiante. As pessoas mais idosas, que residem em lares ou instalações de cuidados a idosos, são uma população reconhecida com alto risco de queda, devido a muitos indivíduos terem: déficit nas atividades de vida diárias, disfunções cognitivas e visuais, serem polimedicamentados, sentirem dor, terem incontinência urinária assim como redução dos níveis de força e equilíbrio ${ }^{42}$.

O fisioterapeuta apresenta um papel de suma importância na prevenção de quedas em idosos através da orientação para a realização de atividades físicas, alongamentos, fortalecimento muscular, treino de marcha e equilíbrio, buscando a manutenção ou melhoria da capacidade funcional, redução das incapacidades e limitações. Independente se o programa de exercícios é feito em grupo ou individualmente, o mesmo promove vários benefícios no controle de quedas $\mathrm{s}^{9,43}$.

A realização do fortalecimento muscular no indivíduo idoso pode potencializar o fator determinante para a melhora da independência e da qualidade de vida, além de ser melhor indicador para a prevenção do risco de quedas. O fortalecimento muscular leva a melhora da função do idoso, e quando associado a outros exercícios funcionais leva a ganho de equilíbrio. O fortalecimento pode ser feito com uso de pesos ou faixas elásticas, que darão resistência ao movimento ${ }^{44}$.

Os exercícios terapêuticos na água (hidroterapia) têm papel importante na prevenção, manutenção e melhora da funcionalidade do idoso. A água oferece suporte e minimiza o estresse biomecânico nos músculos e articulações. As propriedades físicas da água irão auxiliar ainda mais os idosos, na movimentação das articulações, na flexibilidade, na diminuição da tensão articular (baixo impacto), na força, na resistência, nos sistemas cardiovascular e respiratório, no relaxamento, na eliminação das tensões mentais, entre outros ${ }^{15}$.

O Tai Chi tem efeitos significativos no equilíbrio e na força dos músculos extensores dos joelhos em idosos. $\mathrm{O}$ treinamento proprioceptivo específico em idosos acarreta diminuição de oscilações que representem riscos de queda, diminuição do tempo para percorrer determinada distância e, consequentemente, melhora no equilíbrio estático e dinâmico de idosos, com um grau de dependência ${ }^{44}$.

A prática regular de exercícios físicos promove melhoras significativas nos aspectos equilíbrio, flexibilidade, funcionalidade e aumento da resistência muscular, reduzindo o risco de quedas e consequentemente quebrando o ciclo de quedas. $O$ tipo de exercício físico mais eficaz na prevenção das quedas nos idosos é o treino de equilíbrio. Mudanças moderadas a elevadas do equilíbrio, após um programa centrado no treino de equilíbrio, conduzem a um significativo efeito protetor na redução de quedas. $O$ exercício físico é mais eficaz se prolongado no tempo e de maior intensidade - maior número de horas e pelo menos duas vezes por semana ${ }^{11,43}$.

Os idosos ficam expostos a vários fatores de risco, principalmente em suas residências. Portanto, é importante a realização de ações que diminuam estes fatores. Ações e adaptações recomendadas são: evitar camas muito altas; usar sapatos apropriados e dispositivos de apoio para marcha (bengala, andador); não encerar pisos; instalar corrimãos nas escadas e rampas; providenciar iluminação adequada para a noite; instalar, no banheiro, vaso sanitário mais alto e barras de apoio próximo ao chuveiro e ao vaso sanitário; os tapetes devem ser antiderrapantes; concertar calçadas e degraus quebrados ${ }^{45}$.

\section{Conclusão}

A queda representa um risco alto para a saúde do idoso, mesmo não sendo uma consequência do envelhecimento, ela sinaliza que há uma fragilidade ou indício de alguma doença 
aguda presente. Além dos problemas médicos, as quedas apresentam custo social, econômico e psicológico enormes, aumentando a dependência e a institucionalização, sendo uma das principais causas de incapacitação e óbito.

É de extrema importância que os profissionais de saúde voltados para a área de geriatria e gerontologia, estudem e conheçam o tema "quedas" nessa população, pois, o tratamento das consequências geradas pelas quedas e a prevenção das mesmas, são ligados a fatores como controle de medicamentos que podem ser fatores de risco; educação em saúde para o idoso e seus familiares; exercícios e atividades físicas como protocolo de tratamento; acessibilidade e orientação e manutenção da capacidade funcional.

Concluímos que programas de prática de exercícios físicos, treinos de equilíbrio e propriocepção, cinesioterapia, exercícios terapêuticos na água e até mesmo o Tai Chi, são abordagens eficazes para prevenir e para tratar alterações advindas de quedas em idosos. É necessário que o profissional faça uma avaliação única e individual no idoso, olhando-o de forma geral, para tratar não só as consequências e fatores de risco que geram as quedas, mas também, trabalhar o neuropsicomotor daquele paciente, visando sempre a sua capacidade funcional e qualidade de vida.

\section{Referências}

1. Cunha P, Pinheiro LC. O papel do exercício físico na prevenção das quedas nos idosos: uma revisão baseada na evidência. Rev Port Med Geral Fam. 2016;32(2):96-100.

2. Oliveira GG, Knorst MG, Blattner CN, Urbanetto JS, El-Kik RM. Perfil de risco de queda de idosos internados em uma unidade de internação geriátrica de um hospital universitário do município de Porto Alegre. RBCEH. 2016; 13(3):309-22.

3. Gai J. Fatores associados a quedas em mulheres idosas residentes na comunidade. Rev Assoc Med Bras. 2010;56(3):327-32.

4. Abreu DROM, Azevedo RCS, Silva AMC, Reiners AAO, Abreu, HCA. Fatores associados à recorrência de quedas em uma coorte de idosos. Ciênc. saúde coletiva. 2016, 21(11):3439-3446.

5. Fhon JRS, Wehbe SCCF, Vendruscolo TRP, Stackfleth R, Marques $S$, Rodrigues RAP. Quedas em idosos e sua relação com a capacidade funcional. Rev. Latino-Am. Enfermagem. 2012;20(5).

6. Abreu DROM, Novaes ES, Oliveira RR, Mathias TAF, Marcon SS. Internação e mortalidade por quedas em idosos no Brasil: análise de tendência. Ciênc. saúde coletiva. 2018;23(4):1131-41.
7. Dátilo GMPA, Cordeiro AP. Envelhecimento humano: diferentes olhares. Marília: Oficina Universitária; 2015.

8. Alves RLT, Silva CFM, Pimentel LN, Costa IA, Souza, ACS; Coelho, LAF. Avaliação dos fatores de risco que contribuem para queda em idosos. Rev. Bras. Geriatr. Gerontol. 2017;20(1):59-69.

9. Sherrington C, Fairhall NJ, Wallbank GK, Tiedeman A, Michalef ZA, Howard K. et al. Exercise for preventing falls in older people living in the community (Protocol). Cochrane Database of Systematic Reviews. 2016;11. CD012424.

10. Beck AP, Antes DL, Meurer ST, Benedetti TRB, Lopes $M A$. Fatores associados às quedas entre idosos praticantes de atividades físicas. Texto Contexto Enferm. 2011;20(2):280-6.

11. Brito TA, Fernandes MH, Coqueiro, RS, Jesus, CS. Quedas e capacidade funcional em idosos longevos residentes em comunidade. Texto \& Contexto Enferm. 2013;22(1):43-51.

12. Silva FA, Matos MIB, Esteves LSF. Avaliação do risco de queda em idosos independentes. Colloq Vitae. 2017;9(1):18-22.

13. Oliveira $T$, Baixinho $C L$, Henriques MA. Risco Multidimensional de queda em idosos. Rev Bras Promoç Saúde. 2018;31(2):1-9.

14. Lima UMF, Carvalho RS, Almeida LM, Costa AP, Oliveira SX, Nóbrega MM. Intervenção fisioterapêutica quanto à prevenção de quedas em idosos. FIP - Faculdade Integrada de Patos, 2018.

15. Lima DA, Cezario VOB. Quedas em idosos e comorbidades clínicas. Revista HUPE, 2014;13(2):30-37.

16. Esquenazi D, Silva SRB, Guimarães MAM. Aspectos fisiopatológicos do envelhecimento humano e quedas em idosos. Revista HUPE. 2014;13(2):11-20.

17. Azevedo LS. A queda no idoso: fatores de risco e prevenção. Faculdade de Medicina da Universidade de Coimbra. Jan. 2015.

18. Franciulli PM, Souza GB, Albiach JF, Santos KCP, Barros LO, Santos NT, et al. Efetividade da hidroterapia e da cinesioterapia na reabilitação de idosos com histórico de quedas. Estud. interdiscipl. envelhec. 2015;20(3):671-86.

19. Álvares LM, Lima RC, Silva RA. Ocorrência de quedas em idosos residentes em instituições de longa permanência em Pelotas, Rio Grande do Sul, Brasil. Cad. Saúde Pública. 2010;26(1):31-40.

20. Freitas TS, Cândido ASC, Fagundes IB. Queda em idosos: causas extrínsecas e intrínsecas e suas con- 
sequências. Revista Enfermagem Contemporânea. 2014;3(1):70-9.

21. Gontijo KCP. Proposta de intervenção na prevenção de quedas dos idosos no ambiente domiciliar. Universidade Federal de Minas Gerais - Formiga. 2011.

22. Lopea RA, Corrêa RD. O impacto das quedas na qualidade de vida dos idosos. ConScientiae Saúde. 2010;9(3): 504-9.

23. Bretan O, Silva Junio JE, Ribeiro, OR, Corrente JE. Risk of falling among elderly persons living in the community: assessment by the Timed up and go test. Braz J Otorhinolaryngol. 2013;79(1):18-21.

24. Maciel A. Quedas em idosos: um problema de saúde pública desconhecido pela comunidade e negligenciado por muitos profissionais da saúde e por autoridades sanitárias brasileiras. Rev Med Minas Gerais 2010; 20(4):5547.

25. Nascimento JS, Tavares DMS. Prevalência e fatores associados a queda em idosos. Texto Contexto Enferm. 2016;25(2):e0360015.

26. Freitas EV. Tratado de geriatria e gerontologia. 3.ed. Rio de Janeiro: Guanabara Koogan; 2013.

27. Santos DO, Monteiro PRS, Dela Cela M. Atuação do fisioterapeuta na prevenção de quedas em idosos hospitalizados. Pós-Graduação em Fisioterapia Hospitalar - EBMSP, 2015.

28. Lacerda CF, Silva LO, Canto RST, Cheik NC. Efeitos da adaptação às próteses auditivas na qualidade de vida, no equilíbrio e no medo de queda em idosos com perda neurossensorial. Int. Arch. Otorhinolaryngol. 2012.;6(2):156-62.

29. Nicolussi AC, Fhon JRS, Santos CAV, Kusumota L, Marques S, Rodrigues RAP. Qualidade de vida em idosos que sofreram quedas: revisão integrativa da literatura. Ciênc. saúde coletiva. 2012;17(3):723-30.

30. Oliveira HML, Rodrigues LFR, Carus MFB, Freire, NSA. Fisioterapia na prevenção de quedas em idosos: Revisão de Literatura. Revista Interdisciplinar de Estudos Experimentais. 2017;9(único):43-7.

31. Ferreira DCO, Yoshitome AY. Prevalência e caraterísticas das quedas de idosos institucionalizados. Rev Bras Enferm. 2010;63(6):991-7.

32. Pereira GN, Morsc P, Lopes DGC, Trevisan MDT, Ribeiro A, Navarro JHN, et al. Fatores socioambientais associados à ocorrência de quedas em idosos. Instituto de Geriatria e Gerontologia. 2019;90(1): 619-90.

33. Rodrigues RMT, Cervanes M. A influência da Fisioterapia na prevenção de quedas no idoso - Revisão
Bibliográfica. Universidade Fernando Pessoa; 2018.

34. Salzbron CA, Barroso MC, Lopes NP, Herber RR, Ribeiro, SJP. Fatores intrínsecos e extrínsecos que ocasionam a queda em idosos. Faculdade Santa Cruz. Curitiba. 2013.

35. Santos F, Encarnação F. Modernidade e gestão da velhice. Centro Regional de Segurança Social do Algarve, 1997.

36. Bento PCB, Rodacki ALF, Homann D, Leite N. Exercícios físicos e redução de quedas em idosos: uma revisão sistemática. Rev Bras Cineantropom Desempenho Hum. 2010;12(6):471-9.

37. Souza ABS, Ribeiro DS. Análise de escalas funcionais para classificação do risco de quedas em idosos. Revista Brasileira de Reabilitação e Atividade Física. 2012;1(1):1-6.

38. Souza LHR. Queda em idosos e fatores de risco associados. Rev. Aten. Saúde. 2017;15(54):55-60.

39. Rezende CP, Gaede-Carrilho MRG, Sebastião ECO. Queda entre idosos no Brasil e sua relação com o uso de medicamentos: revisão sistemática. Cad. Saúde Pública. 2012;28(12):2223-35.

40. Fonseca SC. O envelhecimento ativo e seus fundamentos. São Paulo: Portal Edições; 2016.

41. Schneider ARS. Envelhecimento e quedas: a fisioterapia na promoção e atenção à saúde do idoso. RBCEH. 2010;7(2):296-303.

42. Rezende AAB, Silva, IL, Cardoso FB, Beresford H. Medo do idoso em sofrer quedas recorrentes: a marcha como fator determinante da independência funcional. Acta Fisiatr. 2010;17(3):117-21.

43. Nogueir A, Alberto, M, Cardoso GA, Barreto MAM. Risco de queda nos idosos: educação em saúde para melhoria da qualidade de vida. Revista Práxis. 2012;(8).

44. Rosa TS, Andriollo DB, Ve APZ, Mai CG. Fisioterapia aquática como prevenção de quedas na terceira idade: revisão de literatura. Cinergis. 2013;14(1):25-8.

45. Gasparotto LPR, Falsarella GR, Coimbra AMV. As quedas no cenário da velhice: conceitos básicos e atualidades da pesquisa em saúde. Rev. Bras. Geriatr. Gerontol. 2014;17(1):201-9. 